\section{Fascial sling more effective than Burch colposuspension at reducing stress incontinence}

A recent study comparing the fascial sling to the Burch colposuspension in women with stress incontinence found the sling procedure to be more effective, but with more complications.

Albo and colleagues performed a multicenter, randomized surgical trial of women with urethral hypermobility and pure or stresspredominant incontinence. Of the 655 women randomly assigned to either the sling procedure $(n=326)$ or the Burch procedure $(n=329)$, 520 completed the study. Patients were assessed at baseline and at 6 weeks, 3, 6, 12, 18 and 24 months following surgery. Success was evaluated using a pad test, diary, cough and Valsalva stress test, self-reported symptoms, and re-treatment rates. At 24 months, the women in the sling group had significantly higher overall success rates $(47 \%$ vs $38 \%$, $P=0.01)$ and stress incontinence-specific success rates (66\% vs $49 \%, P<0.001)$, compared with those in the Burch group.

Adverse events were significantly more common in the women who underwent the sling procedure, compared with the Burch group (63\% vs $47 \%, P<0.001)$. The main adverse events were urinary tract infections, and the adverse event rates were similar between the two groups when urinary tract infections were excluded. Voiding difficulty was also more common in the sling group $(P<0.001)$, as was postoperative urge incontinence $(P=0.04)$. Treatment satisfaction was higher in the sling group $(P=0.02)$.

The authors recommend that clinicians discuss these tradeoffs with patients making treatment decisions, and highlight that complete resolution of symptoms is unlikely.

Original article Albo ME et al. (2007) Burch colposuspension versus fascial sling to reduce urinary stress incontinence. New Engl J Med 356: 2143-2155

\section{Metastatic CDC can be safely treated with gemcitabine plus platinum salt}

Collecting duct carcinoma $(C D C)$ is a rare renal cancer that is usually metastatic at initial presentation. Radical nephrectomy, radiotherapy and immunotherapy are all ineffective for treatment of metastatic cases, so chemotherapy has been suggested. The standard first-line therapy for metastatic urothelial cancer (gemcitabine plus cisplatin) holds promise because the histology of CDC is similar to that of urothelial carcinoma, but prospective evidence of a response in CDC cases was lacking.

Oudard et al. conducted a prospective, phase II study evaluating the efficacy and safety of gemcitabine plus platinum salt in 23 chemotherapy-naive patients with metastatic CDC; 19 had $\geq 2$ metastases. Patients (median age 65 years [range 18-74 years]) were enrolled from six French centers between 2001 and 2005. Drugs (gemcitabine $1,250 \mathrm{mg} / \mathrm{m}^{2}$ on days 1 and 8 , cisplatin or carboplatin [for renally insufficient patients] on day 1) were administered every 3 weeks for a median six cycles; adjustments depended on the level of toxic effects.

Objective response rate was $26 \%$ (one patient had a complete response and five had a partial response). The safety profile of the therapy was acceptable; there were no treatment-related deaths and toxicity was mainly hematological.

Gemcitabine plus platinum salts should be considered as standard first-line therapy for patients with metastatic CDC.

Original article Oudard S et al. (2007) Prospective multicenter phase II study of gemcitabine plus platinum salt for metastatic collecting duct carcinoma: results of a GETUG (Groupe d'Etudes des Tumeurs Uro-Génitales) study. J Urol 177: $1698-1702$

\section{Quality of life after prostate cancer therapy is affected by the treatment method}

The various treatments for localized prostate cancer have different effects on patient quality of life (QOL). Litwin et al. conducted a prospective study to evaluate the effects on health-related QOL of patients with early-stage prostate cancer who were treated with radical prostatectomy (RP), external beam radiotherapy (EBRT), or brachytherapy.

Of the 580 participants in the study, 307 underwent radical prostatectomy, 78 were treated with EBRT, and 90 had brachytherapy. General QOL was evaluated using the Medical Outcomes Study SF-36, and disease-specific QOL was assessed with the UCLA Prostate 